\title{
Distribución espacial de muérdago enano en la Reserva de la Biosfera Mariposa Monarca
}

\section{Spatial distribution of dwarf mistletoe in Monarch Butterfly Biosphere Reserve}

\author{
Nancy Martínez-Martínez ${ }^{\circledR}{ }^{\circledR}$, José Francisco Ramírez-Dávila ${ }^{1 *}{ }^{\circledR}$, Fidel Lara-Vázquez ${ }^{\circledR}$, \\ Dulce Karen Figueroa-Figueroa $^{10}$
}

Martínez-Martínez, N., Ramírez-Dávila, J. F., Lara-Vázquez, F. y Figueroa-Figueroa, D. K. (2021). Distribución espacial de muérdago enano en la Reserva de la Biosfera Mariposa Monarca. Colombia Forestal, 22(2), 65-81.

Recepción: 3 de noviembre 2020

\section{Resumen}

El género Arceuthobium son plantas parásitas de gran impacto ecológico al ocasionar la pérdida de especies forestales; con ello, se ve afectado el hábitat de la mariposa monarca. El objetivo del presente trabajo fue determinar la distribución espacial de muérdago enano en Pinus pseudostrobus, mediante el uso de técnicas de estadística espacial (SADIE y Geoestadística), en 12 parcelas de 4 hectáreas del Santuario Piedra Herrada, ubicado en el Estado de México. Con el Índice SADIE se determinó la distribución espacial; para el caso de la Geoestadística se elaboraron semivariogramas y mapas de distribución espacial, y se realizó la estimación de la superficie infestada. Los resultados mostraron que el muérdago presenta una distribución agregada, ajustándose a modelos estadísticos esféricos y gaussianos. Las infestaciones no se distribuyen en el $100 \%$ debido a que no presenta una infestación uniforme dentro de las parcelas, lo que permitirá dirigir medidas de control en áreas con mayor infestación.

Palabras clave: Geoestadística, haustorios, hospedero, infestación, krigeado, planta parásita.
Aprobación: 7 de abril 2021

\begin{abstract}
The genus Arceuthobium are parasitic plants of great ecological impact by causing the loss of forest species, in which it is affected the habitat of the monarch butterfly. The objective of this work was to determine the spatial distribution of dwarf mistletoe in Pinus pseudostrobus, through the use of spatial statistics (SADIE and Geostatistics), in 12 plots of four hectares, in the Piedra Herrada Sanctuary located in the State of Mexico. With the SADIE index, the spatial distribution was determined, in the case of geostatistics, semivarygrams, space distribution maps and the estimation of the infested area were developed. The results showed that mistletoe presents an aggregate distribution, adjusting to spherical and Gaussian statistical models. Infestations are not distributed at $100 \%$ because there is not an uniform infestation within the plots, which will allow directing control measures in areas with greater infestation.
\end{abstract}

Keywords: Geostatistics, haustoria, host, infestation, Krigeado, parasitic plant.

Universidad Autónoma del Estado de México, México.

jframirezd@uaemex.mx. Autor para correspondencia. 


\section{INTRODUCCIÓN}

La Reserva de la Biosfera Mariposa Monarca se ubica en los Estados de Michoacán y México, y su área la constituye una superficie total de 56259 ha. El bosque predominante es de clima templado con especies como: oyamel (Abies religiosa) y pinos (Pinus L.). La reserva es una zona primordial para la conservación, pues en ella se presenta el fenómeno natural de la migración de la mariposa monarca (Danaus plexippus), quien viaja desde Canadá, pasando por Estados Unidos, para Ilegar a México (Galindo y Rendón, 2005). Por su parte, las especies del género Arceuthobium son conocidas comúnmente como muérdago enano (sus principales hospederos pertenecen a las familias Pinaceae y Cupressaceae), y en la Reserva se encuentra presente una de ellas, el muérdago enano (Arceuthobium globosum Hawksw \& Wiens), plantas hemiparásitas con un gran impacto ecológico y económico para especies del género Pinus. A. globosum se distribuye desde los 1475 a 3990 $\mathrm{m}$ de altitud y constituye uno de los problemas más relevantes en la pérdida de cobertura forestal, después de insectos descortezadores y de incendios forestales (Cházaro et al., 1992; Cibrián et al., 2007; Hawksworth, 1983; Mathiasen et al., 2008; Quiñones y Mathiasen, 2010).

El ciclo de vida del muérdago enano se divide en cuatro fases: dispersión, establecimiento, incubación y reproducción. Estas plantas florecen a partir de 4 a 6 años y sus frutos maduran de 5 a 16 meses, liberando sus semillas en una distancia aproximada de 20 metros; semillas que están cubiertas por una sustancia pegajosa Ilamada viscina que les permite adherirse a cualquier superficie de contacto. Son plantas de 17 a $35 \mathrm{~cm}$ de altura que forman agrupaciones globosas con los tallos de color amarillo claro o amarillo-verdoso. La floración ocurre de junio a mayo y la dispersión de la semilla de junio a octubre del siguiente año. Sus hospedantes principales son: Pinus michoacana Mart, Pinus montezumae Lamb, Pinus leiophyIla Schiede ex Schltdl. \& Cham, Pinus hartwegii
Lindl., Pinus pseudostrobus Lindl., Pinus douglasiana Martínez y Pinus rudis Endl. (deBruyn et al., 2015; Hawksworth et al., 2002).

Estas plantas, al ser pequeñas, dependen completamente de su huésped para satisfacer sus demandas de soporte físico, carbohidratos, agua y sales minerales a través de sus haustorios (raíces modificadas). Así, las infestaciones por muérdago afectan las relaciones hídricas del árbol huésped y el intercambio de gases fotosintéticos. Los síntomas que muestra el hospedero están relacionados con cambios en la forma, reducción de la altura del hospedero, disminución de su volumen con alteración en la calidad de la madera, menor producción de conos, cantidad y calidad de las semillas. Además, provoca la susceptibilidad al ataque de otras plagas y enfermedades, y eventualmente, en infestaciones muy severas, la muerte del huésped. Todos estos síntomas, a su vez, se ven afectados por la edad del árbol, la competencia, la calidad del sitio y el clima (Leimu, 2010; Marias et al., 2014; Roth, 2001; Varo et al., 2016).

Por lo anterior, es necesario saber cómo se distribuye y qué tipo de comportamiento presenta el muérdago enano en la superficie de estudio, a través de mapas de distribución espacial o de infestación y de semivariogramas, resultados con los cuales se pueden generar programas de manejo eficaces y eficientes, pues constituyen una herramienta útil para dirigir las medidas de control hacia zonas donde existe mayor porcentaje de infestación. Estos métodos, al tomar en cuenta las coordenadas geográficas, permiten conocer la ubicación exacta del muérdago dentro de la zona de estudio, y con ello ayudan a optimizar recursos económicos y a generar una reducción en el impacto ambiental por el uso de agroquímicos, ya que las aplicaciones no se realizarían de forma homogénea; además, se pueden realizar acciones de tipo preventivo con las cuales obtener niveles de infestaciones bajas.

Por ello el objetivo del presente trabajo fue determinar la distribución espacial de muérdago enano (Arceuthobium globosum) en Pinus 
pseudostrobus, en el Santuario Piedra Herrada de la Reserva de la Biosfera Mariposa Monarca (Estado de México), mediante el uso de técnicas de SADIE y Geoestadística.

\section{MATERIALES Y MÉTODOS}

\section{Área de estudio}

El presente trabajo se realizó en el Santuario Piedra Herrada de la Reserva de la Biosfera Mariposa Monarca (Estado de México), ubicado a $25 \mathrm{~km}$ de Valle de Bravo, situado en las faldas del Nevado de Toluca, entre las coordenadas geográficas: Latitud $19^{\circ} 11^{\prime} 18^{\prime \prime}$ N Longitud 9957'32.1" W. (Figura 1) Este santuario está constituido por un Bosque con árboles forestales de las especies de oyamel y pino, así como por una gran cantidad de vegetación de especies de flora y fauna endémicas de la región. El paisaje lo componen imponentes montes de más de $3000 \mathrm{~m}$ de altitud. El sitio de muestreo presenta un clima templado húmedo con abundantes Iluvias en verano $(\mathrm{Cw})$ según la clasificación de Köppen.

\section{Tamaño de la muestra}

Para lograr los objetivos, en la zona de estudio se establecieron, de forma aleatoria en toda el área del Santuario, 12 parcelas de 4 hectáreas cada una. Las parcelas presentaron un rango altitudinal de 2800 a 3200 m de altitud; en dichas parcelas se encontraron árboles forestales de las especies: P. pseudostrobus, P. montezumae y P. leiophylla. Sin embargo, para realizar el presente trabajo, sólo se muestrearon árboles forestales de la especie $P$. pseudostrobus con presencia de muérdago enano (A. globosum). Se hizo el muestreo de esta especie porque era la más abundante y la que más se distribuía en toda el área del Santuario.

En cada parcela se estableció una malla rectangular dividida en cuadrantes de 30×30 m cada uno, cubriendo cuatro hectáreas. Veinte (20) cuadrantes fueron tomados al azar y de cada uno se consideraron 6 árboles escogidos de forma aleatoria para un total de 120 árboles. Se cuantificó el número de muérdagos presentes para cada unidad de muestreo, y cada árbol muestreado fue debidamente etiquetado con una banda plástica y

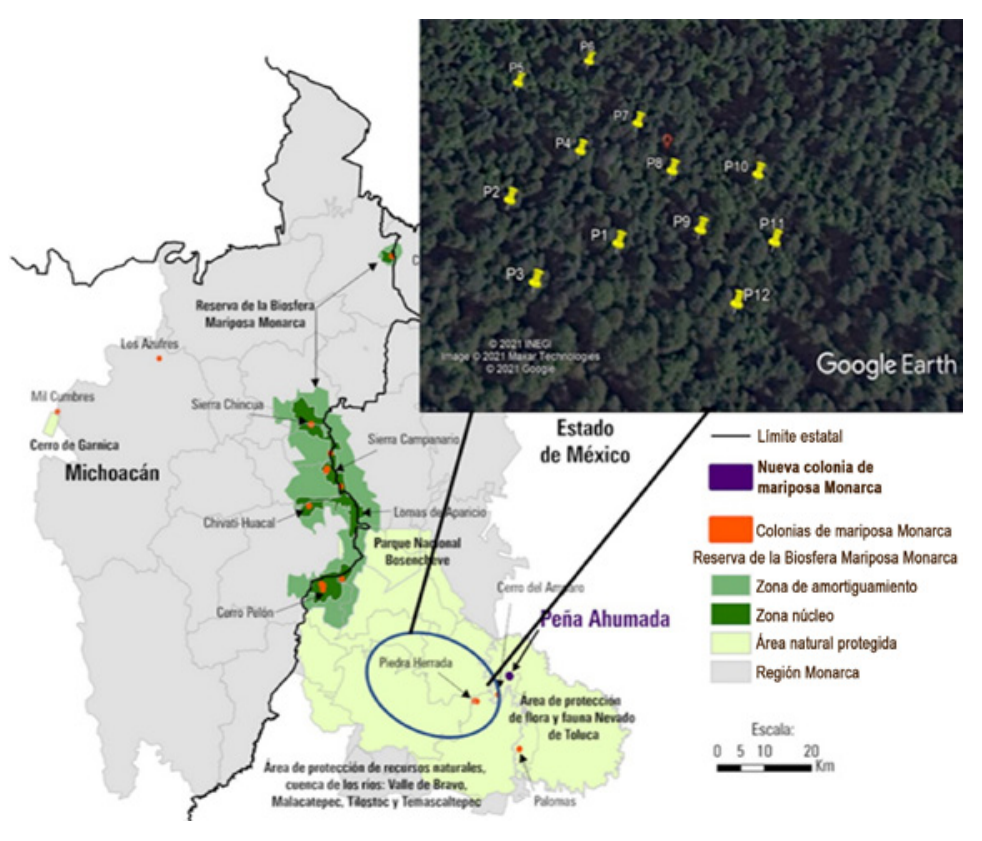

Figura 1. Reserva de la Biosfera Mariposa Monarca y el área de estudio Santuario Piedra Herrada (tomado y modificado de Rendón-Salinas et al., 2018). 
se georreferenció utilizando un DGPS (Differential GPS) (Modelo SPS 351. Timble) para obtener su ubicación espacial (Ramírez y Porcayo, 2010). Debido a la altura a la que se encuentra el A. globosum, el conteo e identificación se hizo con el uso de binoculares (Modelo Vanta). El período de los muestreos se distribuyó entre agosto y diciembre de 2019 para las primeras 5 parcelas, y de enero a julio de 2020 las 7 parcelas restantes (cada muestreo sólo se llevó a cabo por una única vez). Se realizó una exploración estadística de los datos originales de las poblaciones de A. globosum para cada muestreo con la finalidad de determinar la normalidad de los datos. Se utilizó para tal hecho el coeficiente de asimetría y la prueba de Curtosis. Con ello se logró determinar que en todos los casos la distribución de los datos era normal.

\section{Estadística espacial (SADIE)}

Se empleó SADIE (Spatial Analysis by Distance Indices) para explorar si una variable sigue un patrón espacial determinado, ya sea homogéneo, heterogéneo o aleatorio, y esto se basó en la utilización de índices de distancia. Además, se llevó a cabo una prueba de permutaciones donde los valores de las variables fueron distribuidos al azar en el espacio. Se utilizó el índice de la distancia para la regularidad $\left(I_{a}\right)$ y el índice $J_{a}$. El índice $I_{a}$ es una medida del patrón espacial general para un grupo de datos en un área de estudio determinada. El índice $J_{a}$ estima el número de focos de agrupamientos de la población de muérdagos índices que determinan el patrón espacial total (Perry, 1995).

Para la detección de agregados en el área de estudio se utilizó el índice de agregación $\left(I_{a}\right)$ que determina la estructura espacial de las poblaciones de muérdago. Cuando $I_{a}>1$ el patrón espacial es agregado, $l_{a}=1$ el patrón espacial es aleatorio y si $I_{a}<1$ el patrón espacial es regular. Como en el caso del índice $I_{a^{\prime}}$ valores de $J_{a}>1$ usualmente indican una muestra agregada, $J_{a}=1$ representa datos especialmente aleatorios y $J_{a}<1$ muestras regulares. De esta manera, los valores del índice $J_{a}$ sirvieron para corroborar los resultados obtenidos con el índice $I_{a}$ (Ramírez y Porcayo, 2009).

\section{Análisis geoestadístico}

Se obtuvo el semivariograma experimental a partir del valor promedio de A. globosum en cada muestreo utilizando el programa variowin 2.2 (Software for spatial data analysis in 2D. Springer Verlag, New York, USA). El valor experimental del semivariograma fue calculado con la siguiente expresión (Isaaks y Srirvastava, 1989; Pannatier, 1996):

$$
\gamma^{*}(h)=\frac{1}{2 N(h)} \sum_{i=1}^{N(H)}\left[z\left(x_{i}+h\right)-z\left(x_{i}\right)\right]^{2}[1]
$$

Donde:

$\mathrm{Y}^{*}(\mathrm{~h})=$ es el valor experimental del semivariograma para el intervalo de distancia $h$.

$N(h)=$ es el número de pares de puntos muestrales separados en el intervalo de distancia.

$\mathrm{z}\left(\mathrm{x}_{\mathrm{i}}\right)=$ es el valor de la variable de interés en el punto muestral $x_{i}$.

$z\left(x_{i}+h\right)=$ es el valor de la variable de interés en el punto muestra $x_{i}+h$.

Estimación de los parámetros del modelo de semivariograma. Una vez obtenido el semivariograma experimental, se realizó el ajuste a algún semivariograma teórico (esférico, gaussiano, exponencial, etc.) (Englund y Sparks, 1988; Moral, 2004). Para ajustar los semivariogramas experimentales a los semivariogramas teóricos en cada parcela se utilizó el programa Variowin.

Validación del modelo teórico. Una vez que los semivariogramas experimentales fueron ajustados a alguno de los modelos teóricos, la validación de dichos modelos se realizó con el procedimiento denominado "validación cruzada" (Hevesi et al., 1992). Los parámetros del modelo a validar Co (efecto pepita), C+Co (meseta) y a (rango o alcance) se fueron ajustando de forma interactiva (prueba y error) hasta la obtención de estadísticos de validación cruzada adecuados. Una vez determinado el parámetro del modelo, este se valida a través de cuatro parámetros estadísticos de 
validación cruzada (Samper y Carrera, 1996). Estos estadísticos son:

- Media de los errores de estimación (MEE): la MEE no debe ser significativamente distinta de 0 (prueba de $\mathrm{t}$ ), en cuyo caso indicaría que el modelo de semivariograma permite el cálculo de estimadores no sesgados.

- Error cuadrático medio (ECM): un modelo de semivariograma se considera adecuado si, como regla práctica, el valor del estadístico es cercano a cero (Hevesi et al., 1992).

- Error cuadrático medio adimensional (ECMA): la validez del modelo se satisface si ECMA está comprendido entre los valores $1 \pm 2(2 / \mathrm{N}) \quad 0,5$ (Ramírez et al., 2013).

- Otro estadístico adicional que consiste en que el valor de la varianza de los errores sea menor a la varianza muestral.

Nivel de dependencia espacial. Se calculó con el fin de determinar el grado de relación entre los datos de muestreo. El valor se obtiene al dividir el efecto pepita entre la meseta y expresando en porcentaje su resultado. Si el resultado es menor a $25 \%$ el nivel de dependencia espacial es alta, si se encuentra entre $26 \%$ y $75 \%$ el nivel de dependencia espacial es moderada y si es mayor al $76 \%$ el nivel de dependencia es bajo (Cambardella et al., 1994; Ramírez y Porcayo, 2009).

Elaboración de mapas. Una vez que los modelos de los semivariogramas correspondientes fueron validados, se utilizó el método geoestadístico denominado "krigeado ordinario" para la elaboración de mapas de densidad de las poblaciones de A. globosum. Se utilizó dicho método para obtener la estimación de la densidad de esta parásita en puntos donde no se había contabilizado el muérdago y así mismo obtener las estimaciones insesgadas correspondientes con valores asociados en puntos que no fueron muestreados. Cada estimación obtenida fue representada en forma de mapa mediante el uso del programa Sufer 9 (2009).

El cálculo de la superficie infestada. Los cálculos de superficie infestada y no infestada se realizaron con base en mapas de densidad después de validar los semivariogramas (Sánchez et al., 2011). El cálculo de infestación se realizó para cada una de las parcelas de estudio.

\section{RESULTADOS}

Análisis espacial por índices de distancia (SADIE). Los datos obtenidos con la aplicación de los índices de distancia (SADIE) permitieron conocer el índice $I_{a} y J_{a}$. Para el caso del índice $I_{a}$ el valor más alto, 1.72, se registró en la parcela ocho y el más bajo, 1.35, en la parcela dos. En todos los casos el índice $I_{a}$ fue significativamente superior a uno (Tabla 1), lo cual define una distribución agregada de la población de A. globosum en las parcelas de estudio. En lo referente al índice $J_{a}$ el valor mayor, 1.24, se dio en la parcela nueve y el más bajo, 1.07, en la parcela dos. El índice $J_{a}$ en todas las parcelas también fue superior a 1. Estos resultados confirman la agregación de las poblaciones de muérdago enano dentro de las parcelas y permiten determinar que la distribución espacial de las poblaciones de A. globosum se concentró en diferentes centros de agregación en las 12 parcelas de estudio.

La distribución que presenta $A$. globosum en las parcelas se puede apreciar en los mapas generados. En ellos se observan varios y diferentes focos de agregación distribuidos en ciertas zonas de las parcelas (Figura 3). Los resultados obtenidos por el método de geoestadística corroboran los datos obtenidos por los índices de distancia SADIE.

Con la ayuda de la geoestadística y los datos obtenidos de los muestreos fue posible la modelación espacial y el mapeo de las poblaciones de $A$. globosum. Los datos se presentaron mediante mapas de infestación para cada parcela. Así mismo, se estableció el porcentaje de superficie infestada y no infestada y los semivariogramas (Figura 2). La mayoría de los muestreos se ajustaron a modelos teóricos, en donde dos de las doce parcelas (1 y 9) se ajustaron al modelo gaussiano y el resto se ajustaron al modelo esférico (Tabla 2). 
Tabla 1. Valores de los índices $I_{a}$ y $J_{a^{\prime}}$ y sus posibilidades $P_{a}$ y $Q_{a}$ en la población de A. globosum.

\begin{tabular}{ccccc}
\hline Parcela & $\boldsymbol{I}_{\mathbf{a}}$ & $\boldsymbol{P}_{\mathbf{a}}$ & $\boldsymbol{J}_{\boldsymbol{a}}$ & $\boldsymbol{Q}_{\boldsymbol{a}}$ \\
\hline 1 & 1.46 & $0.009^{\mathrm{s}}$ & 1.10 & $0.175^{\text {ns }}$ \\
2 & 1.35 & $0.011^{\mathrm{s}}$ & 1.07 & $0.133^{\text {ns }}$ \\
3 & 1.70 & $0.005^{\mathrm{s}}$ & 1.13 & $0.178^{\text {ns }}$ \\
4 & 1.48 & $0.003^{\mathrm{s}}$ & 1.20 & $0.195^{\text {ns }}$ \\
5 & 1.51 & $0.013^{\mathrm{s}}$ & 1.19 & $0.156^{\text {ns }}$ \\
6 & 1.67 & $0.012^{\mathrm{s}}$ & 1.09 & $0.129^{\text {ns }}$ \\
7 & 1.49 & $0.010^{\mathrm{s}}$ & 1.11 & $0.167^{\text {ns }}$ \\
8 & 1.72 & $0.005^{\mathrm{s}}$ & 1.23 & $0.231^{\text {ns }}$ \\
9 & 1.60 & $0.016^{\mathrm{s}}$ & 1.24 & $0.249^{\text {ns }}$ \\
10 & 1.57 & $0.011^{\mathrm{s}}$ & 1.12 & $0.170^{\text {ns }}$ \\
11 & 1.52 & $0.004^{\mathrm{s}}$ & 1.08 & $0.211^{\text {ns }}$ \\
12 & 1.61 & $0.008^{\mathrm{s}}$ & 1.14 & $0.207^{\text {ns }}$ \\
\hline
\end{tabular}

Nota: ns: no significativo al $5 \%$; s: significativo al 5\%.

El valor de efecto pepita fue igual a cero en todas las parcelas. Dicho valor es indicativo de que la escala de muestreo utilizada en el presente trabajo fue la adecuada y el error de muestreo fue mínimo (Tabla 2).

En todos los modelos se presentó un alto nivel de dependencia espacial para cada una de las parcelas (Tabla 2). Los valores de rango se ubicaron entre $7.0 \mathrm{~m}$ y $20.8 \mathrm{~m}$, correspondientes a las parcelas 5 y 11 respectivamente, siendo el rango la distancia máxima hasta la cual existe relación espacial entre los datos (Tabla 2).

Los parámetros estadísticos de validación cruzada (Tabla 3) permitieron confirmar los valores de los modelos ajustados al hallarse dentro de los rangos de aceptación matemática, es decir, el ajuste del modelo es adecuado encontrándose dentro del rango permisible. Ahora bien, en los mapas elaborados con el método del krigeado ordinario (Figura 3) se pueden distinguir los diferentes

Tabla 2. Parámetros (efecto pepita, meseta y rango) de los modelos ajustados a los semivariogramas de A. globosum y el porcentaje de la superficie infestada y no infestada en cada una de las parcelas.

\begin{tabular}{llcccccccc}
\hline $\mathbf{P}$ & Modelo & Varianza & Pepita & Rango & Meseta & $\begin{array}{c}\text { Pepita/meseta } \\
(\mathbf{\%})\end{array}$ & D.E. & S.I. (\%) & $\begin{array}{c}\text { S.N.I. } \\
(\%)\end{array}$ \\
\hline 1 & Gaussiano & 0.02 & 0 & 8.97 & 0.026 & 0 & Alta & 56 & 44 \\
2 & Esférico & 0.07 & 0 & 9.66 & 0.025 & 0 & Alta & 80 & 20 \\
3 & Esférico & 7.04 & 0 & 7.7 & 6.74 & 0 & Alta & 78 & 22 \\
4 & Esférico & 16.96 & 0 & 9.8 & 14.79 & 0 & Alta & 81 & 19 \\
5 & Esférico & 0.79 & 0 & 7 & 0.61 & 0 & Alta & 55 & 45 \\
6 & Esférico & 2002.12 & 0 & 17.59 & 131.2 & 0 & Alta & 85 & 15 \\
7 & Esférico & 1995.22 & 0 & 12.8 & 120 & 0 & Alta & 92 & 8 \\
8 & Esférico & 671.71 & 0 & 17.6 & 129.6 & 0 & Alta & 98 & 2 \\
9 & Gaussiano & 363.62 & 0 & 12 & 307.1 & 0 & Alta & 86 & 14 \\
10 & Esférico & 28.87 & 0 & 16 & 69.75 & 0 & Alta & 74 & 26 \\
11 & Esférico & 144.67 & 0 & 20.8 & 13.05 & 0 & Alta & 87 & 13 \\
12 & Esférico & 1005.38 & 0 & 18.4 & 17.43 & 0 & Alta & 47 & 53 \\
\hline
\end{tabular}

Nota: P: Parcela; D.E: Dependencia Espacial; S.I: Superficie Infestada; S.N.I: Superficie no Infestada. 
Tabla 3. Valores de los estadísticos de la validación cruzada de los semivariogramas obtenidos en los muestreos de A. globosum: media de los errores de estimación (MEE), error cuadrático medio (ECM) y error cuadrático media adimensional (ECMA).

\begin{tabular}{cccccccc}
\hline Parcelas & $\begin{array}{c}\text { Tamaño } \\
\text { muestra }\end{array}$ & $\begin{array}{c}\text { Media } \\
\text { muestral }\end{array}$ & $\begin{array}{c}\text { Varianza } \\
\text { muestral }\end{array}$ & MEE & $\begin{array}{c}\text { Varianza de } \\
\text { los errores }\end{array}$ & ECM & ECMA \\
\hline 1 & 121 & 0.09 & 0.02 & $0.07^{\mathrm{ns}}$ & 0.01 & 0.11 & 1.13 \\
2 & 121 & 0.08 & 0.07 & $0.11^{\mathrm{ns}}$ & 0.05 & 0.13 & 1.09 \\
3 & 121 & 2.14 & 7.04 & $0.10 \mathrm{n}^{\mathrm{s}}$ & 3.92 & 0.09 & 1.1 \\
4 & 121 & 5,53 & 16.96 & $0.14^{\mathrm{ns}}$ & 10.32 & 0.1 & 1.07 \\
5 & 121 & 0.69 & 0.79 & $0.08^{\mathrm{ns}}$ & 0.51 & 0.12 & 1.11 \\
6 & 121 & 119.78 & 2002.12 & $0.11^{\mathrm{ns}}$ & 1951.34 & 0.11 & 1.09 \\
7 & 121 & 340.93 & 1995.22 & $0.13^{\mathrm{ns}}$ & 12628.1 & 0.06 & 1.12 \\
8 & 121 & 122.41 & 671.71 & $0.10^{\mathrm{ns}}$ & 439.02 & 0.14 & 1.1 \\
9 & 121 & 41.09 & 363.62 & $0.12^{\mathrm{ns}}$ & 317.18 & 0.1 & 1.14 \\
10 & 121 & 8.44 & 28.87 & $0.06^{\mathrm{ns}}$ & 22.95 & 0.08 & 1.13 \\
12 & 121 & 22.33 & 144.67 & $0.09^{\mathrm{ns}}$ & 128.65 & 0.12 & 1.07 \\
\hline
\end{tabular}

Nota: ns: diferencia no significativa al $5 \%$.

focos o grupos de agregación de las poblaciones de A. globosum en las parcelas estudiadas. El color rojo indica la mayor cantidad de muérdagos contabilizados en cada caso; por lo contrario, el color blanco indica la ausencia de dicha parásita en la parcela. Para el caso de la parcela 1 hay diferentes focos de agregación en la zona sureste de la parcela; en el caso de la parcela 2 los focos de agregación están dispersos en casi toda el área de la parcela (Figura 3). En el caso de la parcela 5 se presenta infestación en las zonas suroeste y noreste, quedando la zona centro libre de muérdago.

El porcentaje de superficie infestada por $A$. globosum en todas las parcelas no alcanzó el 100 $\%$, pues se presentan parcelas con mayor y menor superficie infestada. La superficie menos infestada se encontró en la parcela 12, con $47 \%$, y la de mayor porcentaje de infestación fue la 8 , con un $98 \%$ (Tabla 2).

\section{DISCUSIÓN}

Con los resultados obtenidos mediante el análisis espacial por índices de distancia (SADIE), fue posible distinguir el patrón espacial de tipo agregado que se guarda en las parcelas, por lo que los índices $I_{a}$ y $J_{a}$ corroboran la distribución espacial de las poblaciones de A. globosum. El índice $I_{a}$ determina una distribución agregada y el índice $J_{a}$ indica que estos se encuentran en diferentes centros de agregación. La cantidad de centros de agregación encontradas en las parcelas se pueden observar en los mapas (Figura 3; Acosta et al., 2017; Ramírez et al., 2013). El conocimiento de la distribución espacial de A. g/obosum dentro de las parcelas permite focalizar estrategias de manejo adecuado hacia puntos específicos de infestación, y por ende, respecto al ahorro económico, debido a que el método a utilizar solo se aplica en zonas con presencia de infestación. 

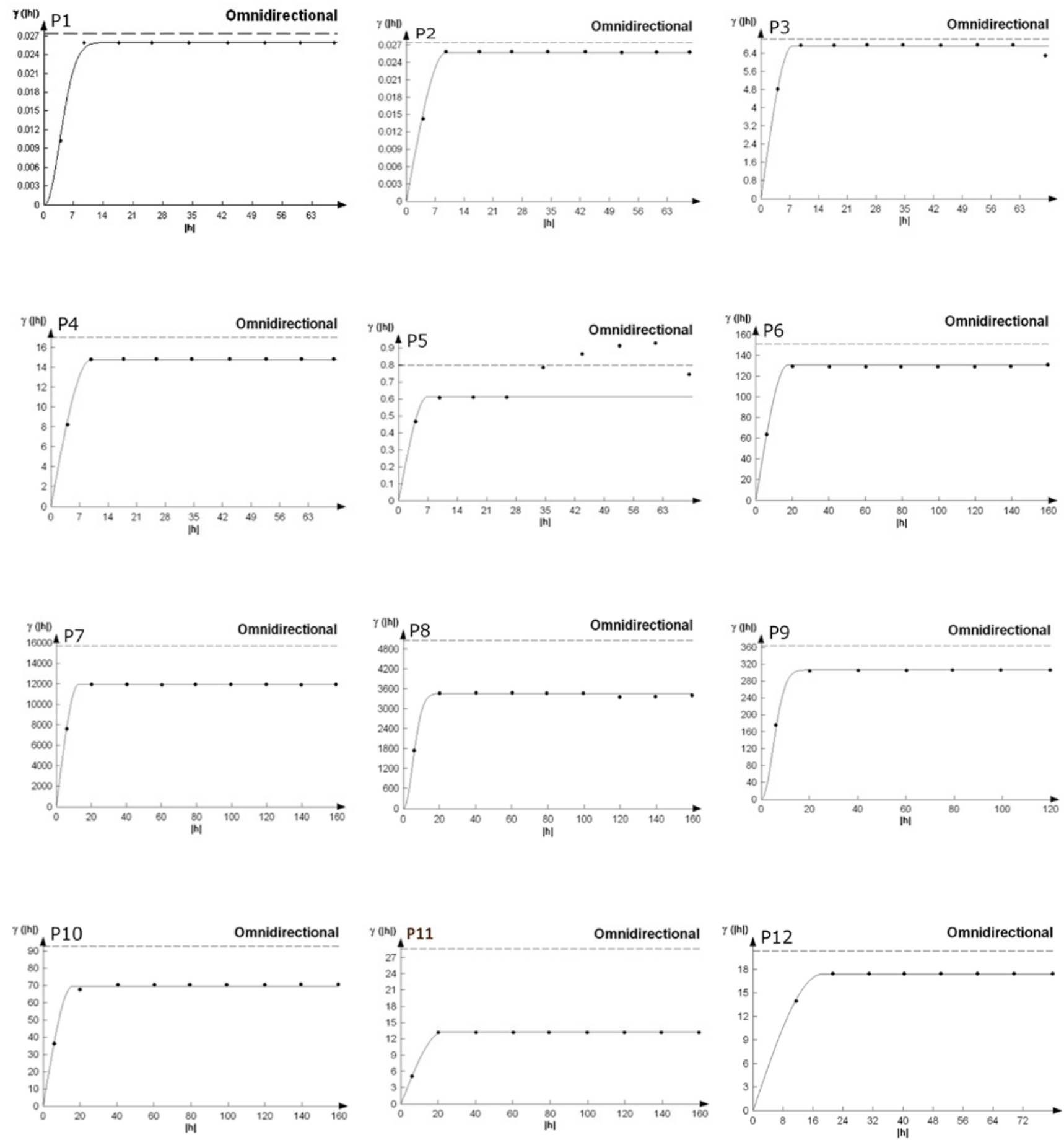

Figura 2. Semivariogramas de la distribución espacial de A. globosum en el Santuario Piedra Herrada para las 12 parcelas estudiadas. 

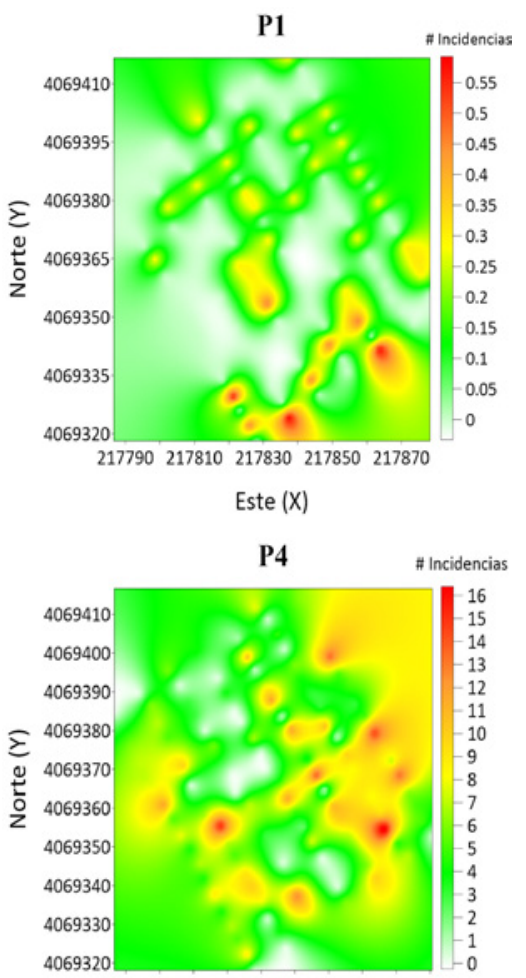

217790217810217830217850217870

Este $(\mathrm{X})$
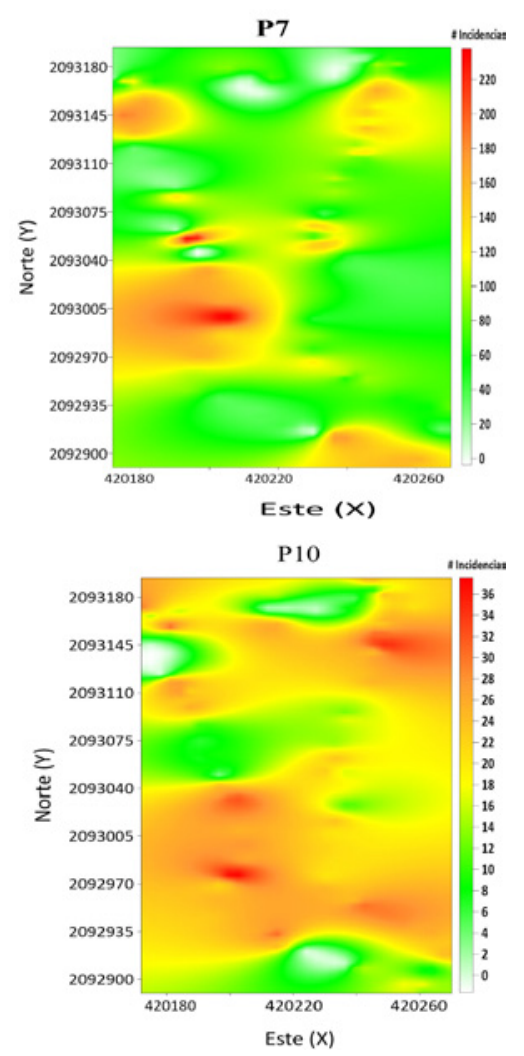
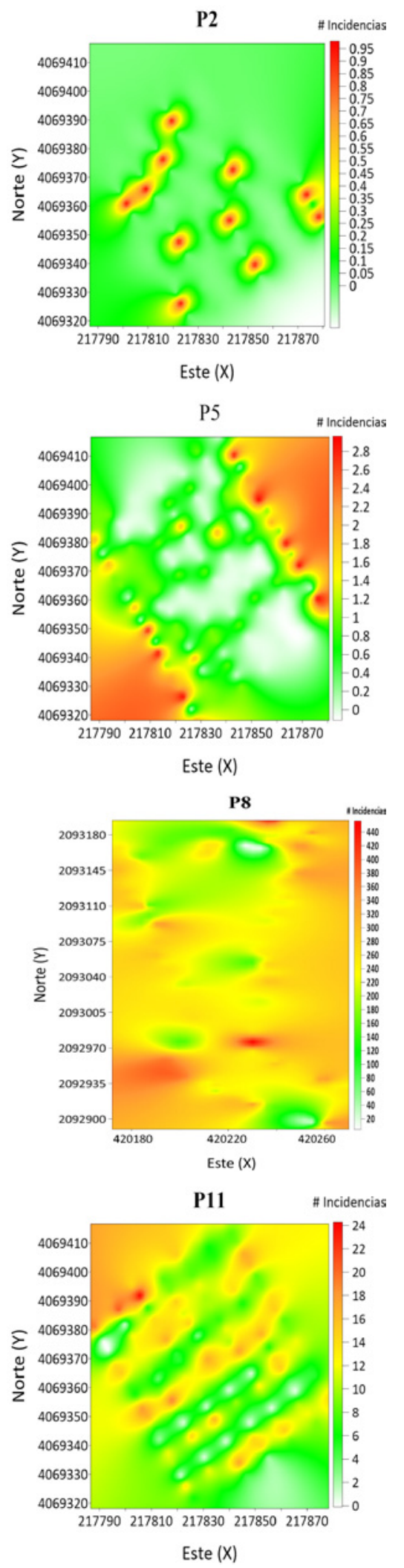

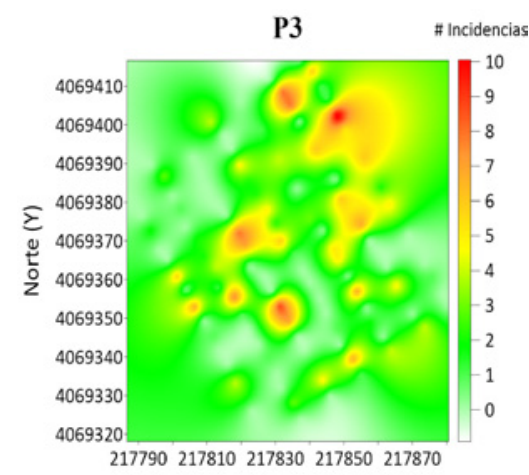

Este (X)
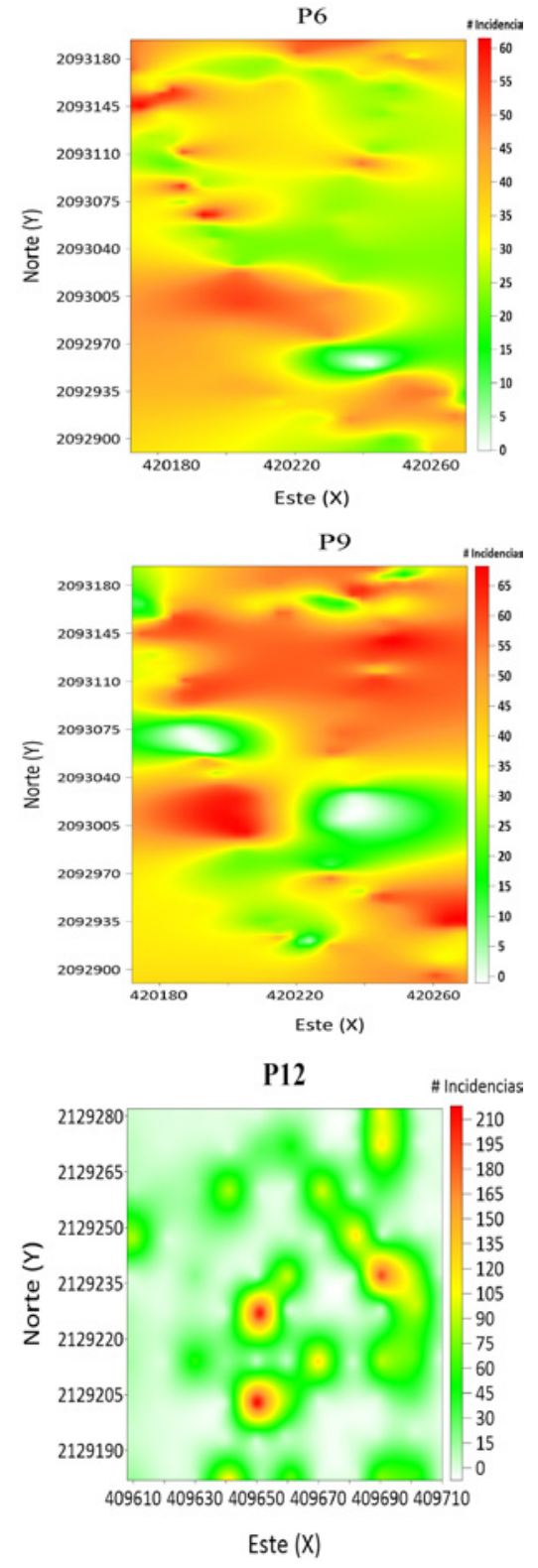

Figura 3. Mapas de densidad de las poblaciones de A. globosum en el Santuario Piedra Herrada. 
Los índices de distancia SADIE se han utilizado con éxito en otros estudios para establecer la distribución espacial de organismos biológicos como insectos, plantas parásitas y enfermedades. Ramírez y Porcayo (2009), por ejemplo, realizaron estudios de la distribución espacial del muérdago enano (Arceuthobium sp.) en el Nevado de Toluca utilizando el método de índice de distancias SADIE. En sus investigaciones concluyeron que el uso de esta metodología permite determinar el comportamiento espacial de las poblaciones de muérdago enano, y dichos resultados se pueden corroborar con ayuda de la geoestadística.

Por su parte, Solís y Suzán (2014), en su estudio sobre el análisis de la distribución espacial de muérdago (Phoradendron californicum Nutt) en el sur del Desierto Sonorense, aluden que los resultados de los índices de agregación obtenidos por SADIE varían dependiendo del tamaño del cuadrante. El estudio menciona que a mayor tamaño o área los índices incrementan y es posible observar un patrón agregado más fuerte. Es probable que la diferencia en el tamaño de las áreas de muestreo no permita ver la agregación en los sitios más pequeños, lo cual impide encontrar agregación en las poblaciones. Por lo anterior, habría que determinar si el tamaño de los cuadrantes en realidad juega un papel importante para determinar el nivel de agregación de A. globosum. Esta metodología no se llevó a cabo en este estudio; sin embargo, sería muy interesante analizar el tamaño de los cuadrantes en estudios posteriores.

Ahora bien, para determinar el tipo de distribución espacial de las poblaciones de organismos biológicos existen dos métodos: la estadística clásica (Índice de dispersión, Índice de Green, Poisson y Binomial negativa) y la estadística espacial (Geoestadística y SADIE). Jiménez et al. (2008) a través de la estadística clásica llevaron a cabo el estudio sobre la distribución y mapeo de Curculio elephas Gyllenhal en encina (Quercus ilex L.), de lo cual se obtuvo una distribución agregada del ente biológico estudiado. Sin embargo, con esta metodología no se puede precisar la ubicación de algún tipo de problema fitopatógeno dentro de las parcelas de estudio, ya que los datos que se tomaron no consideraron su localización georreferenciada dentro de la parcela.

En el caso de Ramírez y Figueroa (2013), quienes realizaron estudios sobre modelización y mapeo de la distribución espacial de Bactericera cockerelli Sulc (Hemiptera: Triozidae) en la papa, en el Estado de México, se utilizaron ambas estadísticas, la clásica y la espacial (Geoestadística). Los resultados obtenidos enfatizaron que la estadística clásica es inconsistente para determinar la ubicación física del insecto y la estadística espacial es la más adecuada, pues se pueden generar mapas de densidad para visualizar la distribución espacial de las poblaciones. Así, en el presente trabajo el uso de técnicas geoestadísticas permitió determinar la distribución espacial de A. globosum bajo una estructura agregada en las 12 parcelas.

Los semivariogramas teóricos obtenidos fueron de dos tipos (esférico y gaussiano). Dichos modelos ajustados tienen un $98 \%$ de fiabilidad, por lo que se puede deducir que más del $90 \%$ de la variación total es debida a la dependencia espacial encontrada en la escala de muestreo utilizada. Esto es, más del $90 \%$ de la variación de la distribución de las poblaciones de A. globosum se logró explicar por la estructura espacial establecida con los semivariogramas. Además, las funciones estadísticas permitieron validar los modelos del tipo esférico y gaussiano de ambas parcelas en las diferentes fechas de muestreo (Acosta et al., 2017; Maldonado et al., 2016).

El modelo gaussiano es indicativo de que el comportamiento de agregación de $A$. globosum se da de forma continua dentro de las parcelas, ya que la infestación de esta planta, con respecto a su avance, se da entre los árboles vecinos de P. pseudostrobus. Resultados similares fueron obtenidos por Sánchez et al. (2015) en su estudio sobre el patrón espacial del carbón de la espiga de maíz y por Contreras et al. (2017) en su estudio sobre el comportamiento espacial de Zebra chip y Bactericera cockerelli Sulc (Hemiptera: Triozidae) en Solanum 
tuberosum L. en Valles Altos de México. Ambos concluyen que los semivariogramas ajustados a los modelos gaussianos indican que los focos de infestación, tanto de la enfermedad como del insecto, presentan una expansión continua dentro de la parcela respecto a los puntos muestreados. Esto permite inferir que la existencia de diversos factores ambientales favorece su desarrollo hacia plantas vecinas.

El modelo esférico indica que las poblaciones de $A$. globosum se presentan en mayor cantidad en ciertas zonas de la parcela respecto al resto de puntos considerados en el muestreo y que los centros de agregación son aleatorios. Posiblemente esto se deba al tipo de diseminación, a la exposición del sol o a los vientos predominantes. Estudios realizados por Nafarrate et al. (2018) sobre la modelización y mapeo estacional del índice de área foliar en un bosque tropical seco y Ramírez y Figueroa (2013) sobre la modelización y mapeo de la distribución espacial de Bactericera cockerelli Sulc (Hemiptera: Triozidae) en papa en el Estado de México, determinaron centros de agregación en zonas específicas dentro de las parcelas de estudio.

En las parcelas se presentó un nivel de dependencia espacial alta y un nivel de agregación alto que permiten suponer la existencia de condiciones ambientales favorables al desarrollo y diseminación del muérdago. Los resultados concuerdan con lo obtenido por Ramírez y Porcayo (2010) en su estudio comparativo de la distribución espacial del muérdago enano (Arceuthobium sp.) en la ladera Norte del Parque Nacional Nevado de Toluca, México. También con lo obtenido por Arce et al. (2016), quienes determinaron un nivel alto de agregación sobre factores bióticos asociados a la distribución espacial de Psittacanthus calyculatus (DC.) G. Don en un bosque tropical caducifolio del centro de México. Por otro lado, Queijeiro y Cano (2015) sugieren que aparte de los factores ambientales se deben tomar en cuenta los disturbios antropogénicos, ya que estos favorecen una mayor incidencia y rebrotes severos posteriores en las zonas. Por lo anterior se tendría que determinar que tanto favorecen los factores ambientales al desarrollo y la expansión de esta parásita hacia zonas en las que se han presentado disturbios antropogénicos, ya que quizás actuando sobre ellos se pueda reducir su presencia en estas zonas. Así, será posible incorporar métodos de control para disminuir el uso de agroquímicos que en algunos casos resultan ser muy costosos y dañinos.

Además de indicar las zonas en las que se encuentra distribuida la plaga, los mapas de infestación también determinan el porcentaje de superficie infestada en cada parcela de estudio. Por ello, se pudo comprobar que la infestación en las parcelas no se distribuye en el $100 \%$, habiendo zonas de poca infestación, lo cual permite dirigir de manera eficaz acciones de control. Estos resultados concuerdan con lo reportado por Ramírez et al. (2011) en el estudio sobre Estabilidad Espacio Temporal de la Distribución del Carbón de la Espiga del Maíz y por Arce et al. (2016) en su estudio sobre factores bióticos asociados a la distribución espacial de muérdago Psittacanthus calyculatus (DC.) G. Don en un bosque tropical caducifolio de México, pues en ninguno de los casos la infestación alcanzó el $100 \%$ del área de estudio, con lo cual se pueden dirigir de forma precisa, eficaz y eficiente las diferentes medidas de control sobre las zonas específicas, generando beneficios económicos y ambientales al aplicar los herbicidas o los controles mecánicos en estas zonas.

En el presente estudio se encontró una distribución agregada dentro de las parcelas, donde se observaron infestaciones altas a menor altitud $y$ pendiente. Estos resultados concuerdan con lo reportado por Varo et al. (2016) en la caracterización de infestación por muérdago enano (Arceuthobium globosum Hawksw \& Wiens subsp. grandicaule) en el bosque de Pinus hartwegii de la cara sur del pico de Orizaba, quienes encontraron que a menor altitud, junto con una menor pendiente, es mayor la infestación, pues la altitud y la pendiente van muy ligados con la incidencia. Sin embargo, Queijeiro y Cano (2015) en su estudio de dinámica temporal de 
la infestación por muérdago enano (Arceuthobium globosum y $A$, vaginatum Willd) en Zoquiapan (Parque Nacional Iztaccihuatl Popocatepetl), detectaron que la infestación es altamente variable entre fechas, especies y sitios debido a que su incidencia se puede ver afectada por sucesos naturales (incendios) o antropogénicos (apertura de nuevos caminos y tala de árboles). Aunque Roxburgh y Nicolson (2005) enfatizan que los patrones de distribución de los muérdagos son afectados tanto por la disponibilidad de hospederos adecuados como por el comportamiento de los dispersores.

Existen alternativas en las que se tiene mayor conocimiento y que pueden proveer métodos efectivos para reducir el impacto de las plantas parásitas. Los métodos de control más empleados incluyen el cultural, el biológico, el químico y el manejo silvicultural. En cuanto al control biológico destacan: Rietman et al. (2005), quienes evaluaron la eficiencia del hongo nativo Neonectria neomacrospora como un agente de control biológico para Arceuthobium tsugense (Rosendahl) G. $\mathrm{N}$ Jones. Alvarado et al. (2010), que identificaron organismos asociados a síntomas de necrosis y defoliación en follaje, frutos y brotes en dos especies de muérdagos verdaderos. Arriola et al. (2012), con su análisis de la presencia de dos especies de insectos que inducen daño sobre el muérdago. Vázquez y Madrigal (2005), al utilizar varias dosis de etephon en el control de Arceuthobium globosum Hawksw. \& Wiens. Y Coria et al. (2010), quienes evaluaron el impacto de tierra de diatomeas sobre Arceuthobium globosum Hawksw \& Wiens subsp. grandicaule en Pinus pseudostrobus Lindl. También se han determinado secuencias específicas de genes de defensa en Abies religiosa (Heredia et al., 2014) como apoyo en el manejo y control del muérdago enano y los descortezadores. En cuanto a las investigaciones de Klutsch et al. (2017) y Klutsch y Erbilgin (2018), se encontró que algunas especies de coníferas resisten a los ataques de plagas y patógenos, lo que ayudaría a disminuir la muerte de árboles y a la conservación de los mismos en las zonas forestales.
La conservación de los bosques es importante para asegurar la continuidad del fenómeno de hibernación de las mariposas y detener la degradación del ambiente natural. La pérdida o deterioro de este hábitat representa un gran riesgo para las poblaciones de mariposas monarca debido a que tan solo la remoción de algunos árboles modifica las propiedades del microclima. La densidad de los árboles y su estructura producen un ambiente específico de temperatura, vientos y otros factores, y sin estas condiciones favorables las mariposas sufren alta mortandad cuando se presentan heladas seguidas por lluvias, lo que provoca un gran desequilibrio ecológico. Dicha conservación, entonces, proporciona beneficios económicos (turismo) y ambientales dirigidos a la conservación de especies de flora y fauna. Así mismo, para mantener las relaciones con los organismos y su medio (procesos ecológicos), y la conservación de servicios ambientales como la calidad y cantidad de agua, y la prevención de erosión y fijación de carbono. Al disminuir la cantidad de árboles va aumentando la cantidad de dióxido de carbono (que no es totalmente absorbido) y en consecuencia se va almacenando en la atmósfera, ocasionando así varios cambios climáticos: la acidificación del agua, el derretimiento de los polos por el aumento de la temperatura, y esto, a su vez, un incremento del nivel del mar, $y$ en algunas zonas se presentan sequias.

Debido a la importancia ecológica que representan los árboles Marias et al. (2014) realizaron estudios para determinar el impacto del intercambio de gases fotosintético y las porciones de isotopos de carbono(C) y oxigeno(O) estables de celulosa en los anillos de los árboles muy infectados y no infectados. Los resultados determinaron que en árboles infestados se reduce el intercambio de gases fotosintéticos.

El uso de algunos herbicidas en el control del muérdago ocasiona fitotoxicidad al hospedante, a las especies aledañas o al hombre. Algunos herbicidas utilizados son Karmex (Diurón), Esteron 47M (2,4-D), Gramoxone (Paraquat), Fitoamina (2, 4-D), Tierra de diatomeas, Etephon y Glifosato, 
aunque Gramoxone y Fitoamina son fitotóxicos para el hospedante. El químico más empleado a la fecha en México ha sido Etephon, producto promisorio en el manejo y control de Arceuthobium y Phoradendron. Se ha evaluado su efectividad con una dosis de 2500 ppm en diversos hospedantes, comprobando que ocasiona una caída del 100\% de la porción aérea de la planta parásita sin dañar al hospedante. Sin embargo, en ninguno de los casos hay eliminación del sistema endófito, por esta razón se desarrollan nuevos brotes aéreos uno o dos años después del tratamiento, y por lo tanto se reduce la dispersión de la semilla durante un periodo superior a cuatro años (Coria et al., 2010). El caso de la aspersión realizada con equipo terrestre resulta ser muy efectiva, mientras que en forma aérea es ineficaz. Esto se debe a que la cantidad del químico en contacto con el muérdago resulta ser insuficiente para causarle la muerte.

La aplicación del herbicida es influenciada por la etapa fisiológica del muérdago y la época de aplicación. La efectividad es mayor en invierno porque el hospedante está en reposo y el muérdago fisiológicamente activo absorbe el producto químico y reduce el daño a los tejidos del árbol, pero en primavera la efectividad no es del todo segura. Una sola aplicación con dosis adecuada logra resultados favorables que van desde la clorosis de hojas, necrosis, defoliación parcial o total, hasta la abscisión de brotes y finalmente la muerte, pero solo de la parte aérea de la planta, pues las raíces quedan intactas.

Al conocer la superficie infestada visualizada en los mapas es posible llevar a cabo una aplicación dirigida hacia los focos de infestación, y con ello lograr ahorros económicos y una reducción de la contaminación ambiental, al disminuir el uso y la cantidad de herbicidas en el control de esta plaga. En la actualidad, en la comunidad de estudio se hace uso de dos herbicidas, Etephon y Fitoamina (2, 4-D), los cuales (en dosis recomendadas) tienen un efecto contaminante en el suelo, el aire y el agua, así como efectos nocivos para la salud humana. La dosis de estos herbicidas va de 2 a 4 L/Ha., dependiendo del grado de infestación, y su costo promedio por litro es de 20 y 15 dólares respectivamente. De esta manera, si se cuenta con la localización exacta de A. globosum y los porcentajes de infestación, se pueden tener ahorros considerables. En este caso las aplicaciones ya no se realizarían de manera homogénea en las parcelas, con lo que se obtendrían beneficios económicos y ambientales, pues conocer las áreas que necesitan un manejo permitiría generar una estrategia de control más adecuada (Lara et al., 2018).

Por tal motivo es de gran relevancia un estudio como el realizado en este trabajo, ya que da una pauta para justificar el uso de las geotécnicas en el manejo de las poblaciones de A. globosum en zonas boscosas. Además, proporciona información relevante y pertinente a las personas que viven en la zona de estudio y a las autoridades respectivas para un manejo integrado, responsable, preciso y oportuno de las poblaciones presentes en este problema fitosanitario.

\section{CONCLUSIONES}

Las técnicas geoestadísticas y el análisis SADIE permitieron modelar la distribución espacial de A. globosum en P. pseudostrobus en el Santuario Piedra Herrada, presentando un comportamiento agregado en cada una de las parcelas experimentales. Así mismo, se determinó que A. globosum no presenta una infestación uniforme en su distribución, lo que permitirá un manejo más eficiente y focalizado en las poblaciones de esta planta parásita, ya que causa gran impacto ecológico y económico en la pérdida de diferentes tipos de ecosistemas (áreas naturales, plantaciones y parques). Por ende, los resultados obtenidos sientan las bases para elaborar un programa de manejo de A. globosum que pueda ser de utilidad por las autoridades competentes y contribuir a la protección y conservación de la riqueza forestal; con ello se 
intenta garantizar el arribo de la mariposa durante su hibernación y conservar así la biodiversidad y calidad ambiental de la región.

\section{AGRADECIMIENTOS}

Los autores agradecen al Ing. Julio Hernández Gutiérrez por su apoyo incondicional al facilitar la información de campo en la identificación de las zonas con presencia de muérdago.

\section{CONFLICTO DE INTERESES}

Cada uno de los autores declaró no tener ningún inconveniente con el manuscrito.

\section{CONTRIBUCIÓN POR AUTOR}

Todos los autores planearon y diseñaron la investigación, coordinaron y llevaron a cabo el trabajo en campo, analizaron los datos y redactaron el manuscrito.

\section{REFERENCIAS}

Acosta, A. D., Ramírez, J. F., Rivera, R., Figueroa, D. K., Lara, A. V., Maldonado, F. I. y Tapia, A. (2017). Distribución Espacial de Trips spp. (Thysanoptera) y Evaluación de su Control Mediante el Depredador Amblyseius swirskii en el cultivo de aguacate en México. Southwestern Entomologist, 42(2), 435-446.

\section{https://doi.org/10.3958/059.042.0214}

Alvarado, D., Saavedra, L. y Almaraz, A. (2010). Determinación taxonómica de los hongos presentes en las diferentes especies de muérdago I arbolado del Distrito Federal (Informe Técnico). Secretaria del Medio Ambiente y Colegio de Postgraduados.

Arce, I., Suzán, H. y García, O. (2016). Biotic factors associated with the spatial distribution of the mistletoe Psittacanthus calyculathus in a tropical deciduous forest of central Mexico. Botanical Sciences, 94(1), 89-96.

https://doi.org/10.17129/botsci.263

Arriola, V. J., Reséndiz, F., Chaires, M. P., Medellín, R. y Pérez, M. (2012). Insecta asociada a muérdago enano (Arceuthobium spp.) en los parques Nacionales Izta-Popo y La Malinche. Entomología Mexicana, 11(2), 829-834.

Cambardella, C., Moorman, T., Novak, J., Parkin, T., Karlen, D., Turco, R. y Konopka, A. (1994). Field Scale variability of soil properties in central lowa Soils. Soil Science Society of America Journal, 58(5), 1501-1511.

https://doi.org/10.2136/sssaj1994.0361599500 5800050033x

Cházaro, M., Huerta, M., Patiño, R. M., Sánchez, R., Lomelí, E. y Flores, A. (1992). Los muérdagos (Loranthaceae) de Jalisco, parásitas poco conocidas. Ciencia y Desarrollo, 17(102), 70-85.

Cibrián, D., Vázquez, I. y Cibrián, J. (2007). Muérdagos enanos del género Arceuthobium. En D. Cibrián, D. Alvarado y S. García (Eds.), Enfermedades forestales en México (pp. 357-395). Universidad Autónoma de Chapingo.

Contreras, A., Gutiérrez, A. T., Sánchez, J. R., Silva, H. V. y Laguna, A. (2017). Comportamiento espacial de Zebra chip y Bactericera cockerelli (Sulc) (Hemiptera: Triozidae) en Solanun tuberosum L. en Valles Altos de México. Revista de la Facultad de Ciencias Agrarias, 49(1), 175-184.

Coria, V. M., Vázquez, I., Muñoz, H. J. y Villa, J. (2010). Impacto de tierra de diatomeas sobre Arceuthobium globosum Hawksworth y Wiens subsp. Grandicaule en Pinus pseudostrobus Lindl. Revista mexicana de Ciencias Forestales, 1(1), 39-46.

https://doi.org/10.29298/rmcf.v1i1.651

deBruyn, R. A., Paetkau, M., Ross, K. A., Godfrey, D. V. y Friedman, C. R. (2015). Thermogenesis-triggered seed dispersal in dwarf mistletoe. Nature Communications, 6.

https://doi.org/10.1038/ncomms7262

Englund, E. y Sparks, A. (1988). GEO-EAS (Geostatistical Environmental Assessment Software) User's Guide. 
U.S. (Environmental Protection Agency Document EPA/600/488/033). Environmental Monitoring Systems Laboratory. https://cfpub.epa.gov/si/si_public_ record_Report.cfm?Lab=ORD\&dirEntryID=36308

Galindo, C., y Rendon, E. (2005). Danaidas: las maravillosas Mariposas Monarca (Publicación especial No. 1). WWF México-Telcel.

https://www.soymonarca.mx/_pdf/danaidas_maravillosas_mariposas_monarca.pdf

Hawksworth, F. G. (1983). Mistletoes an forest parasites. In: M. Calder y P. Bernhardt (Eds.), The Biology of Mistletoes (pp. 320-329). Academic Press.

Hawksworth, F. G., Wiens, D., y Geils B. W. (2002). Arceuthobium in North America. In: B. W. Geils, J. Cibrián y B. Moody (Eds.), Mistletoes of North American Conifers (General Technical Report RMRSGTR-98) (pp. 29-56). United States Departament of Agriculture/Forest Service.

https://doi.org/10.2737/rmrs-gtr-98

Heredia, R. L., Arzate, A. M., Gutiérrez, G., Santillan, J., Cibrián, D. y Endara, A. (2014). Genes de defensa en Abies religiosa. Botanical Sciences, 92(4), 623-628.

https://doi.org/10.17129/botsci.130

Hevesi, J., Istok, J. y Flint, A. (1992). Precipitation estimation in mountainous terrain using multivariate geostatistics. Part. I: structural analysis. Journal of applied meteorology 31(7), 661-676.

https://doi.org/10.1175/1520-0450(1992)031<066 1:PEIMTU>2.0.CO;2

Isaaks, E. y Srivastava R. M. (1989). An introduction to applied Geostatistics. Oxford University Press.

Jiménez, A., López, M. A., González, J. L, Ocete, M. E. y Soria, F. J. (2008). Distribución espacial y mapeo de Curculio elephas Gyllenhal en encina (Quercus ilex L.). Agrociencia, 12(1), 35-43.

Klutsch, J. G. y Erbilgin, N. (2018). Dwarf mistletoe infection in jack pine alters growth-defense relationships. Tree Physiology, 38(10), 1538-1547. https://doi.org/10.1093/treephys/tpy090

Klutsch, J. G., Najar, A., Sherwood, P., Bonello, P. y Erbilgin, N. (2017). A native parasitic plant systemically induces resistance in Jack Pine to a Fungal
Symbiont of invasive Mountain Pine Beetle. Journal of Chemical Ecology, 43(5), 506-518.

Lara, F., Ramírez, J. F., Rubí, M., Morales, E., Figueroa, D. K., Acosta, A. D. y Rivera, R. (2018). Distribución Espacial de Araña Roja Oligonychus punicae Hirst en el Cultivo de Aguacate, en dos Municipios del Estado de México. Southwestern Entomologist, 43(3), 743-759.

https://doi.org/10.3958/059.043.0320

Leimu, R. (2010). Hábitat quality and population size as determinants of performance of two endangered hemiparasites. Annales Botanici Fennici, 47(1), 1-13.

https://doi.org/10.5735/085.047.0101

Maldonado, F. I., Ramírez, J. F., Rubí, M., Nemiga, A. y Lara, A. V. (2016). Distribución espacial de trips en aguacate en Coatepec de Harinas, Estado de México. Revista Mexicana de Ciencias Agrícolas, 7(4), 845-856.

https://doi.org/10.29312/remexca.v7i4.259

Marias, D. E., Meinzer, F. C., Woodruff, D. R., Shaw, D. C., Voelker, S. L., Brooks, J. R., Lachenbruch, B., Falk, K. y Mackay, J. (2014). Impacts of dwarf mistletoe on the physiology of host Tsuga heterophylla trees as recorded in tree-ring $\mathrm{C}$ and $\mathrm{O}$ stable isotopes. Tree Physiology, 34(6), 595-607. https://doi.org/10.1093/treephys/tpu046

Mathiasen, L., Shaw, C., Nickrent, D. y Watson, D. (2008). Mistletoes: Pathology, Sistematics, Ecology, and Management. Plant Disease, 92(7), 988-1006. https://doi.org/10.1094/pdis-92-7-0988

Moral, F. J. (2004). Aplicación de la Geoestadística en las Ciencias Ambientales. Revista Ecosistemas, 13(1), 78-86.

Nafarrate, A. C., Dupuy, J. M., George, S. P. y Hernández, J. L. (2018). Modelización y mapeo estacional del índice de área foliar en un bosque tropical seco usando imágenes de satélite de alta resolución. Madera y bosques, 24(3), 1-17.

https://doi.org/10.21829/myb.2018.2431666

Pannatier, Y. (1996). VARIOWIN: Software for Spatial Data Analysis in 2D. Springer.

https://doi.org/10.1007/978-1-4612-2392-4 
Perry, J. (1995). Spatial analysis by distance indices. Journal of Animal Ecology, 64, 303-314.

Queijeiro, M. E. y Cano, Z. (2015). Dinámica temporal de la infestación por muérdago enano (Arceuthobium globosum y A. vaginatum) en Zoquiapan (Parque Nacional Iztaccíhuatl Popocatépetl), México. Ciencia UAT, 9(2), 6-14. http://doi.org/10.29059/cienciauat.v9i2.705

Quiñones, S. y Mathiasen, R. (2010). First Report of Arceuthobium blumeri on Pinus ayacahuite and $A$. globosum subsp. globosum on $P$. durangensis from Sinaloa, Mexico. Plant Disease, 94(3), 377. https://doi.org/10.1094/pdis-94-3-0377b

Ramírez, J. F. y Figueroa, D. K. (2013). Modelización y mapeo de la distribución espacial de Bactericera cockerelli sulc (Hemiptera: Triozidae) en papa en el estado de México. Revista Centro Agricola, 40(3), 57-70.

Ramírez, J. F. y Porcayo, E. (2009). Estudios de la distribución espacial del muérdago enano (Arceuthobium sp.) en el Nevado de Toluca, México, utilizando el método de SADIE. Madera y Bosques, 15(2), 93-112.

https://doi.org/10.21829/myb.2009.1521193

Ramírez, J. F. y Porcayo, E. (2010). Estudio comparativo de la distribución espacial de muérdago enano (Arceuthobium sp.) en la ladera norte del Parque Nacional Nevado de Toluca, México. Revista Bosque, 31(1), 28-38.

https://doi.org/10.4067/s0717-9200201000010 0004

Ramírez, J. F., Sánchez, J. R. y de León, C. (2011). Estabilidad espacio temporal del carbón de la espiga del maíz (Sporisorium reilianum) en el Estado de México. Revista Mexicana de Fitopatología, 29(1), 1-14.

Rámirez, J. F., Solares, V. M., Figueroa, D. K. y Sánchez, J. R. (2013). Comportamiento especial de trips (Insecta: Thysanoptera), en plantaciones comerciales de aguacate (Persea Americana Mill.) en Zitácuaro Michoacán, Mexico. Acta Zoologica Mexicana, 93, 545-563. https://doi.org/10.25100/socolen.v43i2.5933
Rendon-Salinas, E., Martínez-Meza, F., Mendoza-Pérez, M., Cruz-Piña, M., Mondragón-Contreras, G. y Martínez-Pacheco, A. (2018). Superficie forestal ocupada por las colonias de Mariposas Monarca en México durante la hibernación de 2018-219 (Reporte inédito). WWF-México.

Rietman, L., Karp, B. y Shamoun, S. (2005). Assesment of neonectria neomacrospora (Anamorph cylindroides) as an inundative biocontrol agent against hemlock dwarf mistletoe. Canadian Journal of Plant Pathology, 27(4), 603-609.

https://doi.org/10.1080/07060660509507261

Roth, L. F. (2001). Dwarf mistletoe-induced mortality in Nortwest Ponderosa Pine growing stock. Western Jour of Applied Forestry, 16(3),136-141.

Roxburgh, L. y Nicolson, S. W. (2005). Patterns of host use in two African mistletoes: the importance of mistletoe-host compatibility and avian disperser behavior. Functional Ecology, 19(5), 865-873. https://doi.org/10.1111/j.1365-2435.2005.01036.x

Samper, F. J. y Carrera, J. (1996). Geoestadística. Aplicaciones a la hidrología subterránea ( $2^{\mathrm{a}}$ ed.). CIMNE (Centro Internacional de Métodos en Ingeniería).

Sánchez, J. R., Ramírez, J. F., González, A. y de León, C. (2011). Spatial distribution of head smut (Sporisorium reilianum) of corn in México. Ciencia e Investigación Agraria, 38(2), 253-263. https://doi.org/10.4067/s0718-162020110002 00011

Sánchez, J. R., Ramírez, J. F., González, A. y de León, C. (2015). Patrón espacial del carbón de la espiga del maíz en diferentes localidades del Estado de México. Revista Mexicana de Ciencias Agrarias, 6(7), 1467-1480.

https://doi.org/10.29312/remexca.v6i7.536

Solís, V. y Suzán, H. (2014). Análisis de la distribución espacial de muérdago (Phoradendron californicum) en el desierto Sonorense. Cactáceas y Suculentas Mexicanas, 59(1), 11-28.

Surfer 9. (2009). Surface Mapping System (Versión Golden Surfer 9.0) [Software de computador]. Golden Software Inc.

https://www.scientec.com.mx/surfer/ 
Varo, R., Ávila, V. y Heredia, J. (2016). Caracterización de infestación por muérdago enano (Arceuthobium globosum subsp. grandicaule) en el bosque de Pinus hartwegii en la cara sur del pico de Orizaba. Teoría y Praxis, 19, 11-31.
Vázquez, I. y Madrigal, S. (2005). Control químico de muérdago enano (Arceuthobium globosum) en regeneración de Pinus pseudostrobus. Revista Ciencia Nicolaita, 41, 68-82.

\section{(c) (i) (2)

